\title{
MiR-142-5p Suppresses Tumorigenesis by Targeting PIK3CA in Non-Small Cell Lung Cancer
}

\author{
Zhao Wang ${ }^{a}$ Zhimin Liu ${ }^{b}$ Xiaojie Fang ${ }^{a}$ Han Yang \\ ${ }^{a}$ Department of Medical Oncology, Sun Yat-sen University Cancer Center; ${ }^{b}$ Department of Gastric and \\ Pancreatic Surgery, Sun Yat-sen University Cancer Center; 'Department of Thoracic Surgery, Sun Yat- \\ sen University Cancer Center, State Key Laboratory of Oncology in Southern China, and Collaborative \\ Innovation Center of Cancer Medicine Guangzhou, China
}

\section{Key Words}

MiR-142-5p • NSCLC • Proliferation • Invasion • Migration • PIK3CA

\begin{abstract}
Background/Aims: Numerous studies have demonstrated that aberrant microRNA (miRNA) expression is involved in human disease including cancer. To date, the potential miRNAs regulating lung cancer growth and progression are not fully identified yet. Methods: In this study, the expression of miR-142-5p was measured in non-small cell lung cancer tissue and cell lines by qRT-PCR. The functional assays including the cell viability, colony formation, cell migration and invasion were performed in miR-142-5p mimic or inhibitor transfected cell lines (in vitro) and the cell tumorigenesis in nude mice (in vivo). The fluorescence ratios of cell viability were recorded using a multi-plate reader (Synergy 2, BioTek, Winooski, VT, USA) and the colonies were counted using an ELIspot Bioreader 5000 (BIO-SYS, Karben, GE). Results: MiR-142-5p was significantly downregulated in non-small cell lung cancer tissue and cell lines compared to normal human lung tissues. Overexpression of miR-142-5p resulted in decreased expression of PIK3CA (phosphatidylinositol-4,5-bisphosphate 3-kinase, catalytic subunit alpha) at both mRNA and protein levels. We found that miR-142-5p overexpression markedly suppressed cell proliferation in vitro and in vivo. Conversely, inhibition of miR-142$5 p$ promoted lung cancer growth. Mechanistic studies showed that PIK3CA was a potential target of miR-142-5p and it mediated reduction of PIK3CA resulting in suppression of PI3K/ Akt pathway. Conclusions: Our results demonstrate that miR-142-5p functions as a growth suppressive miRNA and plays an important role in inhibiting the tumorigenesis through targeting PIK3CA in non-small cell lung cancer.

\section{Introduction}

Non-small cell lung cancer (NSCLC) is one of the most common cancers worldwide [1]. The development and progression of NSCLC is typical of a multistage process, which is

Z.Wang and Z. Liu contributed equally to this work. 
believed to involve the deregulation of genes that are critical to cellular processes such as cell cycle control, cell growth, apoptosis, and cell migration and spreading. In the past decades, studies have focused on investigating the genes and proteins underlying the development and progression of NSCLC [2]. Recently, more and more evidence showed that miRNAs play important roles in regulating NSCLC progression [3].

MiRNAs are endogenous non-coding 20 to 22 nucleotide RNAs that have been identified as post-transcriptional regulators of gene expression [4]. They regulate gene expression at the transcriptional and post-transcriptional level by completely or incompletely binding to the 3'-UTR of their target gene messenger RNA (mRNA) and by repressing the translation or promoting the degradation of the target gene to exert biological functions [5-7]. Increasing evidence showed that miRNAs play pivotal roles in tumorigenic processes including cellular differentiation, proliferation, angiogenesis, cell death, apoptosis, and invasion [8]. MiRNAs are deregulated in several diseases including cancers, where they play important roles by regulating the expression of various tumor oncogenes and suppressors $[9,10]$. MiRNAs will hopefully be applied in clinical cancer diagnosis, therapy, and prognosis [11]. To date, deregulated miRNAs and their roles in NSCLC development have attracted much attention. Some of them, including miR-21, miR-34a, miR-124, miR-148, miR-204, miR221,miR-452, miR-497 and miR-509, et al, have been reported to participate in the initiation and progression of NSCLC and modulate the biological properties of cancer cells [12-25]. However, the role of miR-142-5p in NSCLC tumor development and metastasis has only recently been investigated and remains largely unknown.

In the present study, we investigated the role of miR-142-5p in NSCLC. Our results showed that the expression of miR-142-5p was down-regulated in NSCLC cells and tissues compared to paired adjacent non-tumor bone tissues. Moreover, in vitro experiments proved that miR-142-5p inhibited cell proliferation, migration and invasion in the NSCLC cells. In addition, PIK3CA was identified as a novel direct target gene of miR-142-5p. Our findings suggested that miR-142-5p has a tumor suppressive effect in NSCLC by inhibiting cell proliferation and invasion.

\section{Materials and Methods}

Cell culture and human tissue samples

The human embryonic kidney-derived cell line HEK293T, human NSCLC cells lines (A549, SPCA1, H1066, H460 and H358) and normal lung fibroblast cell line HLF were maintained in Dulbecco's modified eagle's medium (DMEM, Hyclone, Thermo Fisher Scientific, Waltham, MA, USA) supplemented with 10\% fetal bovine serum (FBS) (GIBCO, NY, USA) and streptomycin $(100 \mathrm{mg} / \mathrm{ml})$, penicillin $(100 \mathrm{U} / \mathrm{ml})$. Cultures were incubated at $37^{\circ} \mathrm{C}$ with $5 \% \mathrm{CO} 2$ in a humidified incubator.

Eighty three pairs of human NSCLC tumor tissue and adjacent non-tumor tissue samples were obtained immediately after resection from patients undergoing primary surgical treatment in Sun Yat-sen University Cancer Center, Guangdong, China. The tissue samples were frozen in liquid nitrogen and stored at $-80^{\circ} \mathrm{C}$ until use. Written consent for research purposes was obtained from the patients before tissue collection and the protocol was approved by the Institutional Review Board of Sun Yat-sen University.

\section{Transfection}

Cells were transfected with $20 \mathrm{nmol} / \mathrm{L}$ of miR-142-5p mimic, miR-142-5p inhibitor or nonrelative control (NC) duplex (GenePharma, Shanghai, China) and PIK3CA siRNAs or NC siRNA (Ribobo, Guangzhou, China) using FuGene HD transfection reagent (Roche, Indianapolis, IN, USA) according to the manufacturer's protocol.

RNA extraction and quantitative real-time RT-PCR analysis

Total RNA was extracted from human tissues or cell lines using TRIzol reagent (Invitrogen Life Technologies, Carlsbad, CA, USA) according to the manufacturer's protocol. For miRNA detection, $2 \mu \mathrm{g}$ of small RNA was reverse transcribed to cDNA using miRNA First-Strand cDNA Synthesis kit (Invitrogen) 


\section{Cellular Physiology Cell Physiol Biochem 2017;43:2505-2515 and Biochemistry Published \begin{tabular}{l|l} 
DOI: 10.1159/000484459 & $\begin{array}{l}\text { C } 2017 \text { The Author(s). Published by S. Karger AG, Basel } \\
\text { www.karger.com/cpb }\end{array}$
\end{tabular}}

Wang et al.: MiR-142-5p Suppresses Tumorigenesis in NSCLC

according to the manufacturer's instructions. Quantitative real-time PCR (qRT-PCR) analysis for miR142-5p was performed in triplicate with the SYBR Green PCR Master Mix (Takara, JP) according to the manufacturer's instructions. U6 was used to normalize expression. To detect the target genes, $2 \mu \mathrm{g}$ of total RNA was reverse transcribed to cDNA using oligo (dT) primers and Moloney murine leukemia virus reverse transcriptase (Promega). GAPDH were used to normalize expression. Data analysis was performed using the $2^{-\Delta \Delta C \mathrm{t}}$ method.

\section{Cell viability and colony formation assays}

Cell proliferation was analyzed by Cell Titer-Blue cell viability assay (Promega Corporation, Madison, WI, USA) according to the manufacturer's instructions, and the fluorescence ratios were recorded using a multi-plate reader (Synergy 2, BioTek, Winooski, VT, USA). Cell viability was determined at 1, 2, 3, 4 and 5 days after transfection. In addition, 2000 transfected A549 and SPCA1cells were seeded in fresh 6-well plates in triplicate and maintained in DMEM containing 10\% FBS for 2 weeks. Next, cell colonies were fixed in $20 \%$ methanol and stained with $0.1 \%$ coomassie brilliant blue R250 at room temperature for 15 min. Finally, the colonies were counted using an ELIspot Bioreader 5000 (BIO-SYS, Karben, GE).

\section{Tumorigenicity assays in nude mice}

Male BALB/c nude mice ( 5 to 6 wk of age) were obtained from Shanghai Experimental Animal Center (Shanghai, China). Animal handling and experimental procedures were approved by the Animal Experiments Ethics Committee of Sichuan Medical University. For in vivo tumorigenicity assay, all pyrimidine nucleotides in the miR-142-5p mimic, miR-142-5p inhibitor or NC duplex were substituted by their 2'-0-methyl analogues to improve RNA stability. MiR142-5p mimic or miR-142-5p inhibitor transfected A549 cells $(1 \times 106)$ were suspended in $100 \mathrm{uL}$ PBS and then injected s.c. into left side of the posterior flank of 6 BALB/c nude mice, respectively. NC transfected or nontransfected A549 cells $(1 \times 105)$ were injected subcutaneously into right side of same 12 mice. Tumor growth was examined daily and the tumor volumes were calculated every week using the formula for hemi-ellipsoids: $V=$ length $(\mathrm{cm}) \times$ width $(\mathrm{cm}) \times$ height $(\mathrm{cm}) \times 0.5236$. After 5 weeks, the mice were sacrificed and the tumors were dissected and photographed.

\section{Cell migration and invasion assay}

For the cell migration assay, $2 \times 10^{5}$ transfected A549 cells in serum-free media were seeded in the upper chamber of transwell units (Corning, NY, USA) with $8 \mu \mathrm{m}$ pore size polycarbonate filter under serum free condition. The lower chamber was filled with 500 $\mu \mathrm{L}$ DMEM containing $10 \%$ FBS. After incubation for $24 \mathrm{~h}$, cells on the upper surface of the filter were completely removed by wiping with a cotton swab. Then the filters were fixed with $4 \%$ paraformaldehyde and stained with $0.1 \%$ coomassie brilliant blue R250 for $20 \mathrm{~min}$. The number of cells that migrated through the pores to the lower surface of the filter was counted and analyzed with a digital microscope system (IX81; Olympus). Triplicate samples were acquired and the data were expressed as the average cell number of 5 fields. For the cell invasion assay, the similar protocol of cell migration assay was used except that the transwell units were pre-coated with $200 \mu \mathrm{g} / \mathrm{ml}$ Matrigel (BD Biosciences, San Jose, CA, USA) and incubated overnight. Cells that had invaded the Matrigel and reached the lower surface of the filter were counted.

\section{Western blotting analysis}

Proteins were extracted using a modified RIPA buffer with $0.5 \%$ sodium dodecyl sulfate (SDS) and the proteinase inhibitor cocktail (Complete Mini, Roche). Equal amounts of protein were separated on $10 \%$ SDS-PAGE gel, and then transferred to a PVDF membrane (Immobilon P-SQ, Millipore, Billerica, MA, USA). After blocked with 5\% non-fat dried milk, the membrane was incubated with anti-PIK3CA (Abcam, England) at 1:1000 dilution and anti-GAPDH antibody (Proteintech, Chicago, USA) at 1:20, 000 dilution. After washing with TBST (10 mM Tris, pH 8.0, $150 \mathrm{mM} \mathrm{NaCl}$, and 0.1\% Tween 20), the membranes were incubated with HRP-conjugated goat anti-rabbit antibody or goat anti-mouse antibody (1:10000 dilution, KPL, Gaithersburg, MA,USA) for $2 \mathrm{~h}$ at room temperature. Proteins bands were visualized using ECL reagents (Pierce, Rockford, IL, USA).

\section{Luciferase reporter assay}

The 3'-UTR fragment of PIK3CA (Genbank accession no. NM_006218.3) containing the putative binding sequence was amplified using the primers 5'- AAAGATAACTGAGAAAATGAAAGCTC-3' (forward) 
and 5'-GAAGAAAGCTGACCATGCTGCTATG -3' (reverse). The resulting PCR product was cloned into a firefly luciferase reporter vector (pGL3; Promega Corporation, Madison, WI, USA), and termed pGL3-PIK3CA$3^{\prime}$ UTR. A plasmid that carried mutations in the complementary sites for the seed region of miR-142-5p was generated based on the pGL3-PIK3CA-3'UTR plasmid using a MutanBEST Kit (Takara Bio Inc., Shiga, JP), and termed pGL3-PIK3CA-3'UTR-mut. The correctness of the plasmids was confirmed by sequence analysis.

HEK293T cells were co-transfected pGL3-PIK3CA-3'UTR or pGL3-PIK3CA-3'UTR-mut and the miR142-5p mimic or NC duplex. The cells were collected $48 \mathrm{hrs}$ after transfection, and were analyzed for luciferase activity using the dual-luciferase reporter assay kit (Promega Corporation). The pRL-TK construct (Promega Corporation, Madison, WI, USA) was also transfected as a normalization control.

\section{miRNA target prediction}

TargetScan (http://www.targetscan.org) and MiRanda (http://www.microrna.org/microrna/home. do) was used to in silico predict miRNA targets and conserved sites bound by the seed region of miR-142-5p.

\section{Statistical analysis}

Data were presented as the means \pm standard deviation of at least three experiments. Statistical analysis was performed using SPSS 15.0. A one-way analysis of variance (ANOVA) test, least significant difference (LSD) test, Chisquare test and Student's t test were used for statistical analysis.

\section{Results}

\section{Expression of miR-142-5p is downregulated in NSCLC tissues and cell lines}

To investigate the relevance of miR-142-5p in NSCLC, we first set out to measure its expression in 83 pairs of tumor versus adjacent non-tumor tissues by qRT-PCR. MiR-142-5p was found to be down-regulated in tumor tissues compared to the non-tumor tissues tested (Fig. 1A). In addition, we examined the expression of miR-142-5p in human NSCLC cells lines (A549, SPCA1, H1066, H460 and H358) and in normal lung fibroblast cell line HLF using qRT-PCR. As shown in Fig. 1B, the expression of miR-142-5p was much lower in five NSCLC cell lines than that in HLF.

miR-142-5p inhibits NSCLC cell proliferation, migration and invasion

Cells were firstly transfected with miR-142-5p mimics, inhibitors or negative control, which showed high transfection efficiency (data not shown). Cell viability assay showed that cell proliferation capacity was reduced in miR-142-5p mimics-transfected the A549 and

A

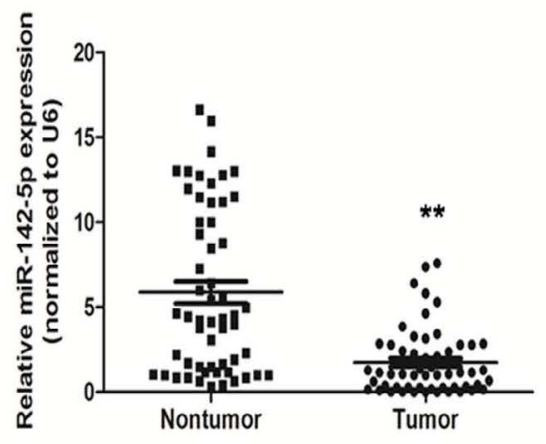

B

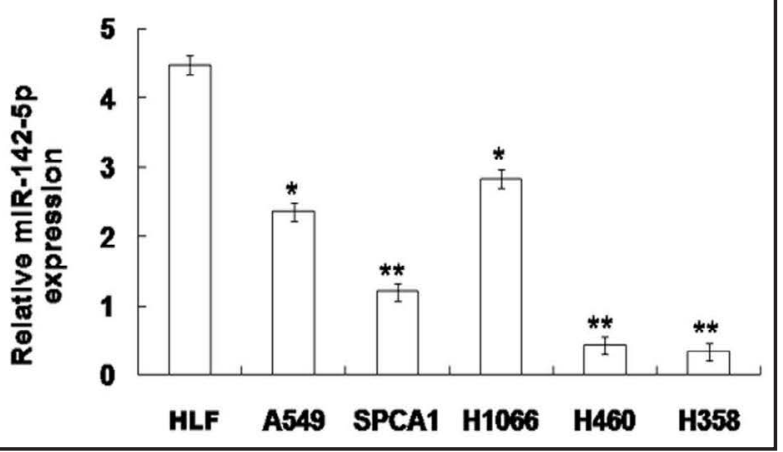

Fig. 1. miR-142-5p is down-regulated in NSCLC. A. The relative expression of miR-142-5p was determined in 83 pairs NSCLC tissues and the corresponding adjacent non-tumor tissues by qRT-PCR. B. The relative expression of miR-142-5p was determined in normal lung fibroblast cell line HLF and NSCLC cells (A549, SPCA1, H1066, H460 and H358) by qRT-PCR. The expression of miR-142-5p was normalized to U6. ${ }^{*} \mathrm{P}<0.05$, ** $\mathrm{P}<0.01$. 
SPCA1 cells compared with either the NC duplex-transfected cells or the untreated cells (Fig. 2A). Conversely, miR-142-5p inhibitor significantly promoted the cell proliferation of the A549 and SPCA1 cells (Fig. 2A). These results indicated that the miR-142-5p significantly suppressed the cell proliferation of the NSCLC cells.

To validate the inhibitory effect of miR-142-5p on cell proliferation, the colony formation assay was performed in A549 and SPCA1 cells transfected with or not, miR-142-5p mimic, miR-142-5p inhibitor or NC duplex. As showed in Fig. 2B, A549 and SPCA1 cells transfected with miR-142-5p mimic displayed much fewer and smaller colonies (471 or 692 colonies) compared with NC duplex transfected (1062 or 1769 colonies) and nontransfected cells (1039 or 1721 colonies), but cells transfected with miR-148a-3p inhibitor displayed much more and larger colonies (1824 or 2168 colonies).

To further confirm the above findings, an in vivo mouse model was used. For the duration of the treatment with miR-142-5p mimic or miR-142-5p inhibitor for 5 weeks, tumor volume curves revealed a significant decrease in growth rates at the 3rd, 4th and 5th week after treatment with miR-142-5p mimic and a significant increase in growth rates at the 4 th and 5 th week after treatment with miR-142-5p inhibitor whereas no significant differences in tumor growth rates were observed between the NC group and the ctrl group (Fig. 2C). These results indicate that introduction of miR-142-5p significantly inhibits tumorigenicity of A549 cells in xenograft nude mouse model.

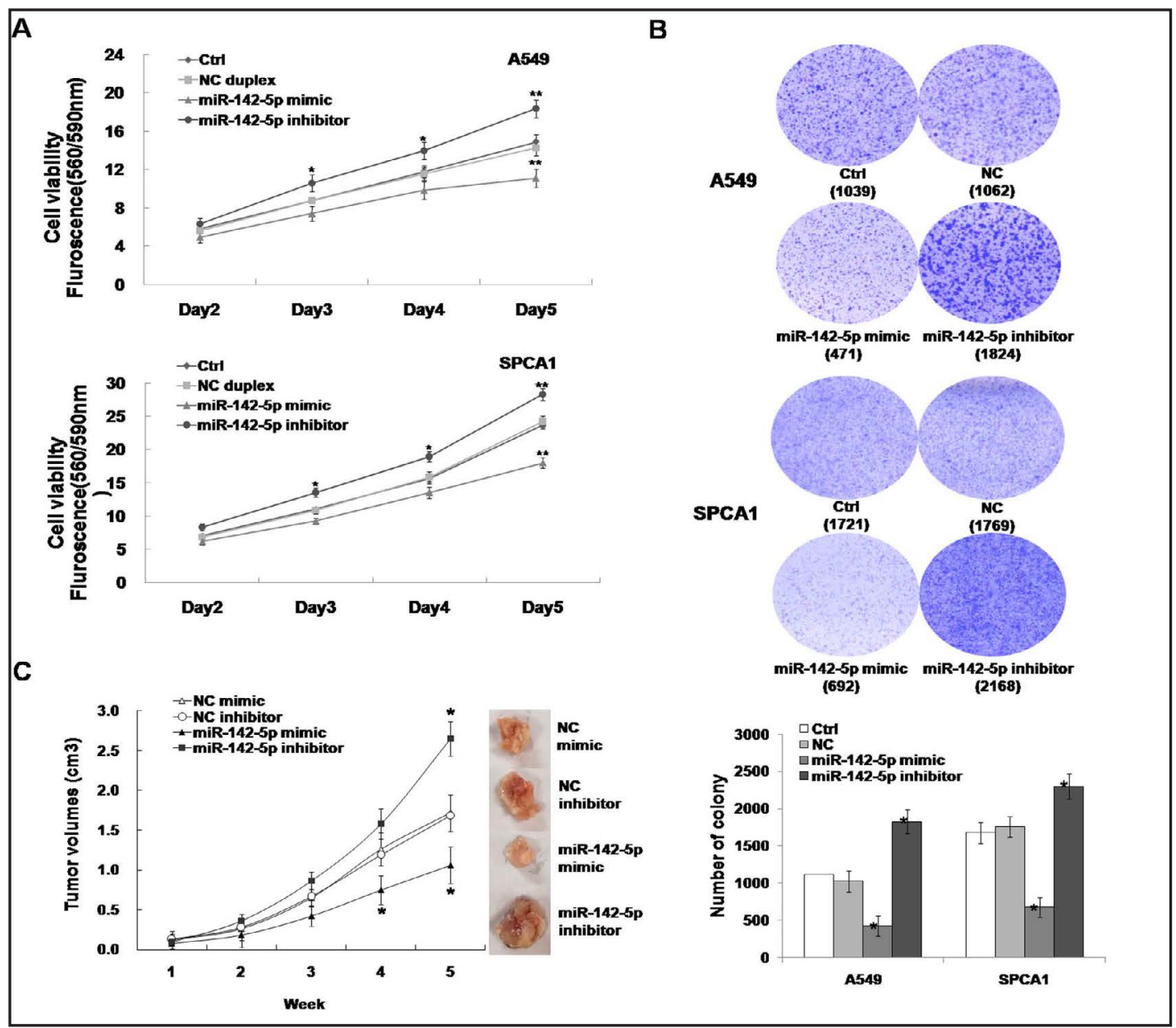

Fig. 2. miR-142-5p inhibits cell proliferation in NSCLC cells. A. Cell viability was determined in A549 and SPCA1 cells. B. Colony formation assay was determined in A549 and SPCA1 cells. C. The effect of miR-142-5p on the tumor growth in xenograft nude mice. Cells were transfected with miR-142-5p mimics, miR-142-5p inhibitors or NC duplex. The data were represented by mean \pm SD. $* P<0.05, * * P<0.01$. 


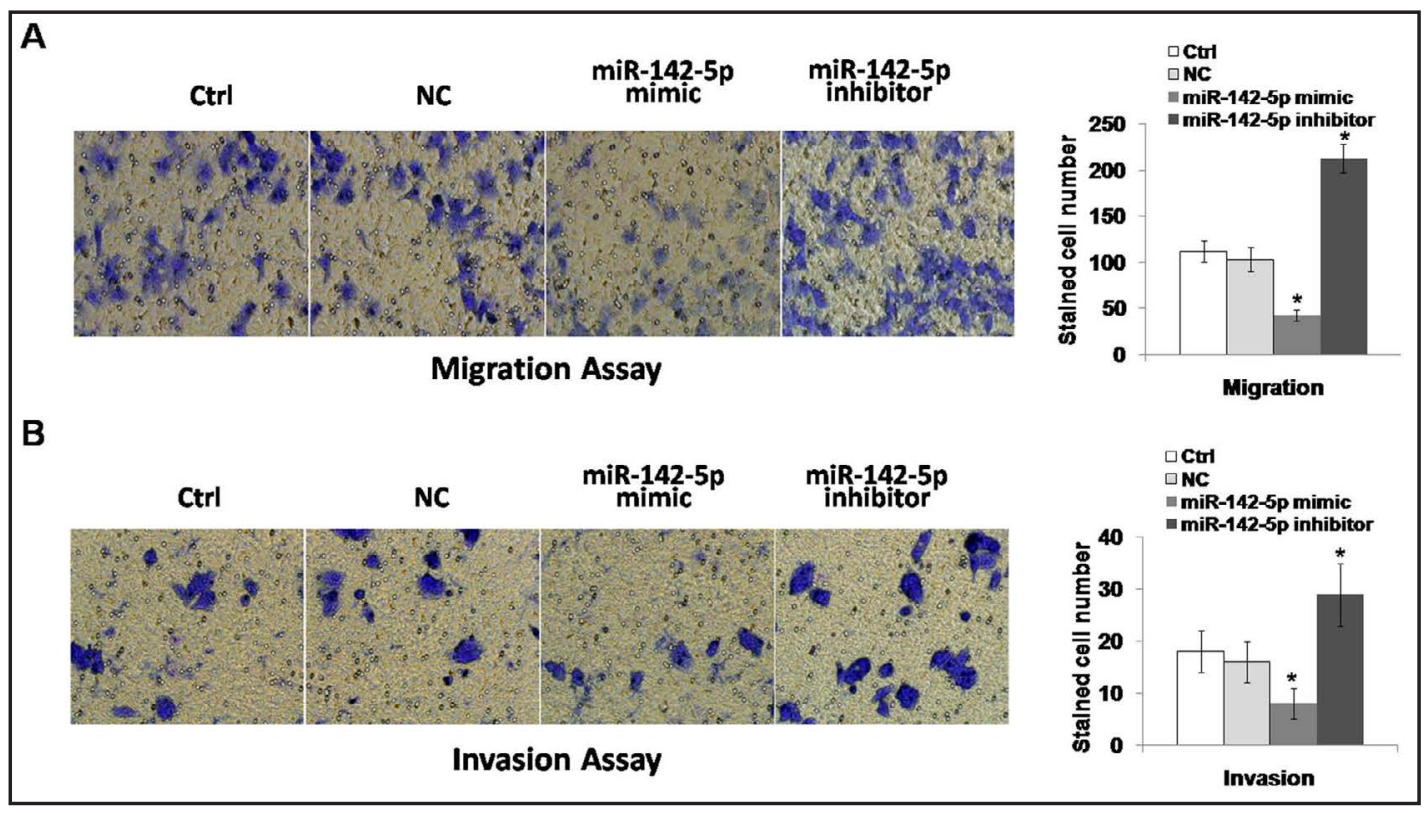

Fig. 3. miR-142-5p inhibits cell migration and invasion in NSCLC cells. A. Cell migration assay was determined in A549 cells. B. Cell invasion assay was determined in A549 cells. Cells were transfected with miR142-5p mimics, miR-142-5p inhibitors or NC duplex. The data were represented by mean \pm SD. ${ }^{*}<0.05$, ** $\mathrm{P}<0.01$.

To further investigate the effect of miR-142-5p on A549 and SPCA1 cells migration and invasion, we transfected the cells with miR-142-5p mimics, inhibitors or negative control and then evaluated migration and invasion ability of NSCLC cells. As the Fig. 3A shown, the cells that were treated with the miR-142-5p mimic were distinctively less migratory than the scrambled control or untreated cells. By contrast, the miR-142-5p inhibitor significantly increased the cell migration of the NSCLC cells. Furthermore, we conducted cell invasion Matrigel assays and then stained the invaded cells to measure the directional invasion ability of the cells after ectopically expressing miR-142-5p in cells. The invasiveness of the cells that were transfected with the miR-142-5p mimic was dramatically decreased compared with the scrambled control and untreated cells. Conversely, the miR-142-5p inhibitor significantly increased the invasiveness of the NSCLC cells (Fig. 3B). These observations suggest that miR$142-5 p$ had an important role in reducing the migration and invasion of NSCLC in vitro.

PIK3CA is a direct target of miR-142-5p

As is well known, miRNAs exert their function through suppressing the expression of their target genes. To better understand the molecular mechanisms of miR-142-5p, we performed bioinformatics analyses the using the miRNA target analysis tools TargetScan and MiRanda in order to predict putative miRNAs binding to the PIK3CA 3'-UTR. According to the analysis, the programs predicted that a binding sequence in the 3'-UTR of PIK3CA was a very good match for the miR-142-5p seed (Fig. 4A). The bioinformatics analysis thus indicated a potential functional link between PIK3CA and miR-142-5p.

Furthermore, we performed a luciferase reporter assay to further confirm whether miR142-5p can directly target the 3'-UTR region of PIK3CA in NSCLC cells. Both the PIK3CA wildtype 3'-UTR containing the miR-142-5p-binding site and a mutated PIK3CA 3'-UTR sequence were cloned into modified pGL-3 luciferase reporter vectors, which were co-transfected into A549 cells together with NC, the miR-142-5p mimic, or miR-142-5p inhibitor. As shown in Fig. 4B, miR-142-5p over-expression significantly reduced the luciferase reporter activity by the PIK3CA 3'-UTR in a consistent manner, and inhibition of miR-142-5p had the opposite effect. However, PIK3CA 3'-UTR luciferase reporter activity was unaffected by point mutations in 


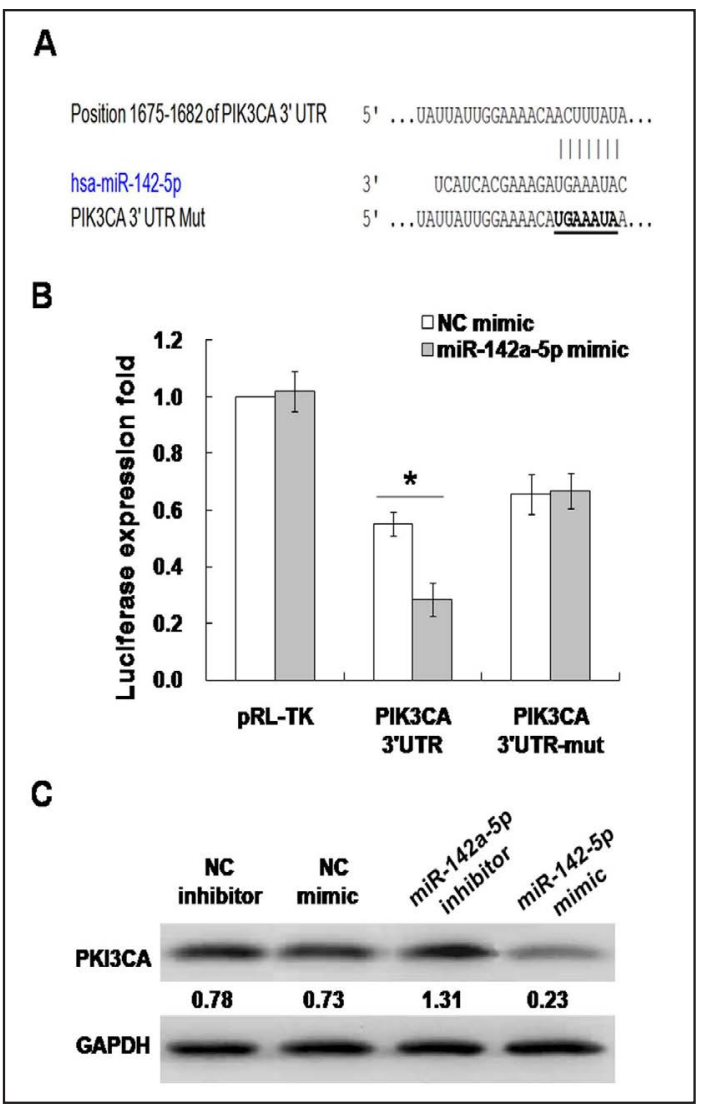

Fig. 4. miR-142-5p directly targets PIK3CA. A. The predicted miR-142-5p binding site within the PIK3CA 3'-UTR and a mutated version generated by site directed mutagenesis are shown. B. Luciferase reporter assay illustrating direct binding of miR142-5p to the WT, but not mutant sequences within the 3'-UTR of PIK3CA. C. The protein expression of PIK3CA was regulated by miR-142-5p in A549 cells. GAPDH was used as a control. The data were represented by mean $\pm \mathrm{SD}$. ${ }^{*} \mathrm{P}<0.05$, ${ }^{* *} \mathrm{P}<0.01$.

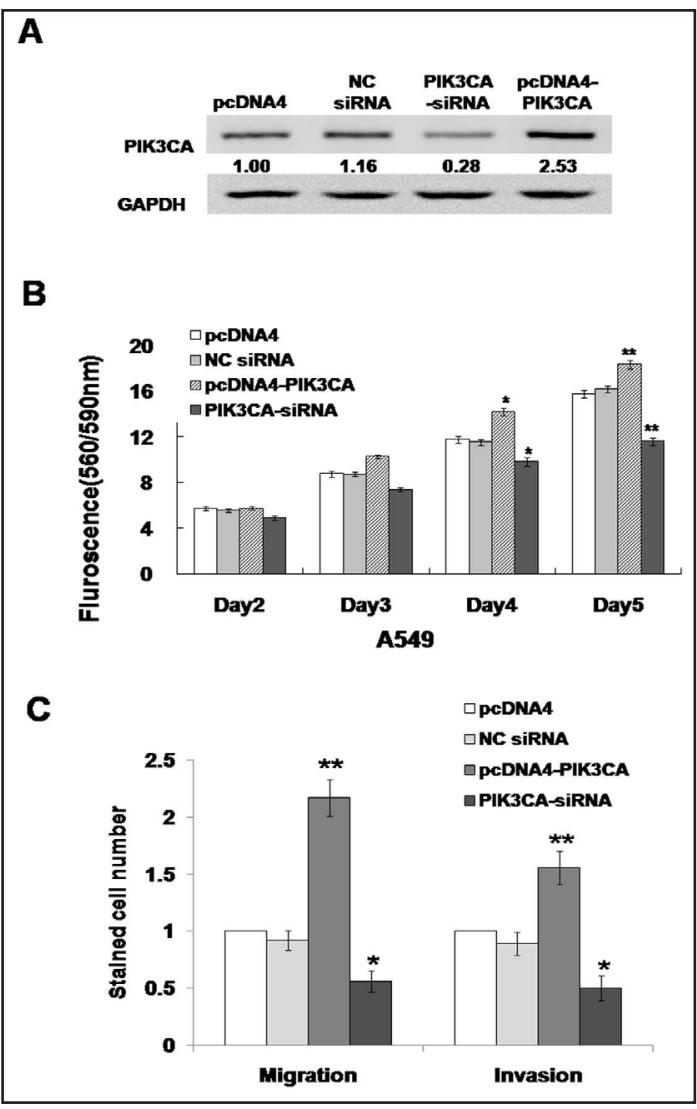

Fig. 5. PIK3CA is involved in miR-142-5p inhibited cell proliferation, migration and invasion in A549 cells. A. The expression of PIK3CA in A549 cells transfected with pcDNA4-PIK3CA, PIK3CA siRNA or pcDNA4, NC siRNA. B. Down-regulation of PIK3CA suppressed cell proliferation in A549 cells. C. Downregulation of PIK3CA suppressed cell migration and invasion in A549 cells. The data were represented by mean $\pm \mathrm{SD} . * \mathrm{P}<0.05, * * \mathrm{P}<0.01$.

the miR-142-5p-binding seed region. Collectively, these data suggest that miR-142-5p may inhibit PIK3CA expression by targeting its 3'-UTR. As predicted, a western blot showed that, at $48 \mathrm{~h}$ after transfection, the enhanced miR-142-5p in A549 cells significantly repressed PIK3CA protein expression compared to cells transfected with a scrambled control. By comparison, down-regulation of miR-142-5p by inhibitors in A549 cells led to a moderate increase in the PIK3CA protein expression (Fig. 4C). Together, these data provide strong evidence that PIK3CA is a specific target of miR-142-5p in NSCLC cells.

\section{PIK3CA is involved in miR-142-5p-inhibitory effect in A549 cells}

Based on the findings above, we hypothesized that miR-142-5p might inhibit NSCLC cell proliferation, migration and invasion by repressing PIK3CA expression. First we transiently transfected PIK3CA siRNA into A549 cells, and qRT-PCR and western blotting confirmed the down-regulation of PIK3CA (Fig. 5A). As shown in Fig. 5B and 5C, PIK3CA silencing significantly suppressed cell proliferation, migration and invasion of A549 cells, which was similar to the phenotype induced by miR-142-5p. 


\section{Discussion}

Accumulating evidence demonstrates that miRNAs have important roles in NSCLC progression and directly contribute to the cell proliferation, avoidance of apoptosis, and metastasis of NSCLC [26-28]. Identifying the miRNAs and their targets that are essential for NSCLC progression may provide promising therapeutic opportunities. Previously, miR142 displays a functional role in various cellular fuctions and disease, including cancer, virus infection, inflammation, and immune tolerance [29]. It has been reported that miR$142-5 p$ suppresses TGF- $\beta$-induced growth inhibition by targeting SMAD3in cancer cells [30]; miR-375 and miR-142-5p regulate several oncogenic genes of TP53, MAPK and Wnt signal pathways in gastric cancer [31]; miR-142-5p inhibits cell growth and induce apoptosis by regulating FOXO in HCC [32]; miR-142-5p, targeting CLDN1, plays an important role in Hashimoto's thyroiditis (HT) pathogenesis [33]; downregulated miR-142-5p significantly reduced cell migration and invasion in HCC [34]. In the current study, we found miR-142-5p was down-regulated in NSCLC cells. In addition, we analyzed the expression of miR-142-5p in NSCLC patients and found that the expression of miR-142-5p was much lower in NSCLC tissues in comparison with paired adjacent non-tumor bone tissues. All of these evidences indicated that miR-142-5p might play a significant part in the development of NSCLC. Then, we performed functional assays. Ectopic expression of miR-142-5p significantly inhibited NSCLC cell proliferation, migration and invasion. These findings suggested that miR-142$5 \mathrm{p}$ might act as a tumor suppressor gene whose down-regulation may contribute to the progression and metastasis of NSCLC.

PI3K is a lipid kinase and generates phosphatidylinositol-3, 4, 5-trisphosphate (PI(3, $4,5)$ P3), which functions as a second messenger for Akt activation [35, 36]. Activated Akt protein modulates cell proliferation through numerous downstream targets, such as Bad, procaspase-9, mammalian target of rapamycin (mTOR), glycogen synthase kinase-3 (GSK-3), Cyclindependent kinase inhibitors, P21 and P27 [37]. PIK3CA is known to be an oncogene component of phosphatidylinositol 3-kinase (PI3K) signaling pathway and is implicated in cell proliferation and carcinogenesis in many human cancers [38, 39]. PIK3CA was significantly upregulated in gastric cancer tissues and miR203 inhibits cell proliferation and invasion via directly targeting and suppressing the PIK3CA expression [40]; miR-1 may play an important role in the pathogenesis of non-small cell lung cancer (NSCLC) by regulating PIK3CA through the PI3K/Akt pathway [41]; miR-19a acted as an oncogenic miRNA regulating renal cancer cell proliferation, migration and invasion by directly targeting PIK3CA [42]; miR-490-5p functions as a tumour suppressor in renal carcinoma by targeting PIK3CA [43]; miR-375 suppressed CRC cell proliferation and colony formation and led to cell cycle arrest by targeting PI3K/Akt signaling pathway [44]; miR-134 and miR-370 could play a fundamental role in suppressing colorectal cancer tumorigenesis by independently targeting EGFR and PIK3CA [45]; miR-124-mediated reduction of PIK3CA, resulting in suppression of PI3K/Akt pathway, plays an important role in inhibiting hepatocellular carcinoma (HCC) tumorigenesis [46]; miR-422a inhibited cell proliferation, invasion, and migration by targeting PIK3CA, and the miR-422a/PIK3CA axis may constitute a potential target for glioblastoma multiforme (GBM) therapy [47]; PIK3CA expression was found to be increased in human papillary thyroid carcinoma (PTC) tissues and was regulated by miR363-3p [48]; MiR-375 functions as a growth-suppressive miRNA and plays an important role in inhibiting the tumorigenesis through targeting PIK3CA in osteosarcoma [49]. In the current study, further investigation was conducted to explore the molecular mechanism by which miR-142-5p suppressed NSCLC cell growth, migration and invasion. In this study, we identified PIK3CA as a functional target of miR-142-5p, and verified the positive effects of PIK3CA on NSCLC cell proliferation, migration and invasion using RNA interference. These results indicated that miR-142-5p might function as a tumor suppressor partly mediated by repressing PIK3CA expression in NSCLC.

\section{KARGER}




\section{Cellular Physiology Cell Physiol Biochem 2017;43:2505-2515 and Biochemistry Published $\begin{aligned} & \text { DOI: 10.1159/000484459 } \\ & \text { (c) } 2017 \text { The Author(s). Published by S. Karger AG, Basel } \\ & \text { www.karger.com/cpb }\end{aligned}$}

Wang et al.: MiR-142-5p Suppresses Tumorigenesis in NSCLC

\section{Conclusion}

The present study provided novel evidence that miR-142-5p function as a tumor suppressor miRNA in NSCLC through repressing PIK3CA expression. Consequently, our findings provided a molecular basis for the role of miR-142-5p/PIK3CA in the progression of human NSCLC and suggested that this miRNA could be a potential target for the treatment of NSCLC in future.

\section{Acknowledgements}

This study was supported by the foundation of 5010 Clinical Trials of Sun Yat-sen University.

\section{Disclosure Statement}

Conflict of interest for all authors - None.

\section{References}

1 Uramoto H, Tanaka F: Recurrence after surgery in patients with NSCLC. Transl Lung Cancer Res 2014;3:242-249.

2 De Marinis F, Bria E, Baas P, Tiseo M, Camerini A, Favaretto AG, Gridelli C: Treatment of Unfit Patients With Advanced Non-Small-Cell Lung Cancer: Definition Criteria According an Expert Panel. Clin Lung Cancer 2015;16:399-405.

3 Villaruz LC, Socinski MA: The Role of Anti-angiogenesis in Non-small-cell Lung Cancer: an Update. Curr Oncol Rep 2015;17:448.

4 Del Vescovo V, Grasso M, Barbareschi M, Denti MA: MicroRNAs as lung cancer biomarkers. World J Clin Oncol 2014;5:604-620.

5 Bartel DP: MicroRNAs: genomics biogenesis mechanism and function. Cell 2004;116:281-297.

6 Schickel R, Boyerinas B, Park SM, Peter ME: MicroRNAs: key players in the immune system, differentiation, tumorigenesis and cell death. Oncogene 2008;27:5959-5974.

7 Tufekci KU, Meuwissen RL, Genc S: The role of microRNAs in biological processes. Methods Mol Biol 2014;1107:15-31.

-8 Silber J, James CD, Hodgson JG: microRNAs in gliomas: small regulators of a big problem. Neuromolecular Med 2009;11:208-222.

-9 Tang JT, Fang JY: MicroRNA regulatory network in human colorectal cancer. Mini Rev Med Chem 2009;9:921-926.

10 Suzuki H, Maruyama R, Yamamoto E, Kai M: DNA methylation and microRNA dysregulation in cancer. Mol Oncol 2012;6:567-578.

11 Zimmerman AL, Wu S: MicroRNAs, cancer and cancer stem cells. Cancer Lett 2011;300:10-19.

12 Wang P, Lv HY, Zhou DM, Zhang EN: MiR-204 suppresses non-small-cell lung carcinoma (NSCLC) invasion and migration by targeting JAK2 Genet Mol Res 2016;15.

-13 Lin L, Tu HB, Wu L, Liu M, Jiang GN: MicroRNA-21 Regulates Non-Small Cell Lung Cancer Cell Invasion and Chemo-Sensitivity through SMAD7.Cell Physiol Biochem 2016;38:2152-2162.

14 Ma T, Zhao Y, Wei K, Yao G, Pan C, Liu B, Xia Y, He Z, Qi X, Li Z, Wang J, Shao Y: MicroRNA-124 Functions as a Tumor Suppressor by Regulating $\mathrm{CDH} 2$ and Epithelial-Mesenchymal Transition in Non-Small Cell Lung Cancer. Cell Physiol Biochem 2016;38:1563-1574.

15 Chen Y, Gao Y, Zhang K, Li C, Pan Y, Chen J, Wang R, Chen L: MicroRNAs as Regulators of Cisplatin Resistance in Lung Cancer. Cell Physiol Biochem 2015;37:1869-1880. 


\section{Cellular Physiology Cell Physiol Biochem 2017;43:2505-2515

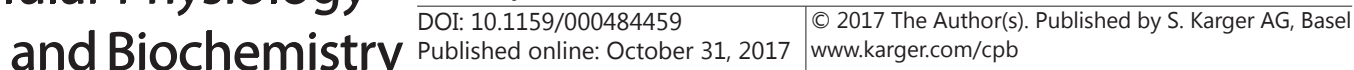

16 Li J, Yu T, Cao J, Liu L, Liu Y, Kong HW, Zhu MX, Lin HC, Chu DD, Yao M, Yan MX: MicroRNA-148a Suppresses Invasion and Metastasis of Human Non-Small-Cell Lung Cancer. Cell Physiol Biochem 2015;37:18471856.

17 Niu X, Liu S, Jia L, Chen J: Role of MiR-3619-5p in $\beta$-Catenin-Mediated Non-Small Cell Lung Cancer Growth and Invasion. Cell Physiol Biochem 2015;37:1527-1536.

18 Zhu X, Fu C, Zhang L, Xu G, Wang S: MiRNAs associated polymorphisms in the 3'UTR of MET promote the risk of non-small cell lung cancer. Cell Physiol Biochem 2015;37:1159-1167.

19 He Z, Xia Y, Pan C, Ma T, Liu B, Wang J, Chen L, Chen Y: Up-Regulation of MiR-452 Inhibits Metastasis of NonSmall Cell Lung Cancer by Regulating BMI1.Cell Physiol Biochem 2015;37:387-398.

20 Shi Y, Liu C, Liu X, Tang DG, Wang J: The microRNA miR-34a inhibits non-small cell lung cancer (NSCLC) growth and the CD44hi stem-like NSCLC cells. PLoS One 2014;9:e90022.

21 Huang C, Ma R, Yue J, Li N, Li Z, Qi D: MiR-497 Suppresses YAP1 and Inhibits Tumor Growth in Non-Small Cell Lung Cancer. Cell Physiol Biochem 2015;37:342-352.

-22 Yin Z, Xu M, Li P: miRNA-221 acts as an oncogenic role by directly targeting TIMP2 in non-small-cell lung carcinoma. Gene 2017;620:46-53.

23 Yan A, Yang C, Chen Z, Li C, Cai L: MiR-761 Promotes Progression and Metastasis of Non-Small Cell Lung Cancer by Targeting ING4 and TIMP2 Cell Physiol Biochem 2015;37:55-66.

24 Deng W, Yan M, Yu T, Ge H, Lin H, Li J, Liu Y, Geng Q, Zhu M, Liu L, He X, Yao M: Quantitative proteomic analysis of the metastasis-inhibitory mechanism of miR-193a-3p in non-small cell lung cancer. Cell Physiol Biochem 2015;35:1677-1688.

25 Ma N, Zhang W, Qiao C, Luo H, Zhang X, Liu D, Zang S, Zhang L, Bai J: The tumor suppressive role of MiRNA509-5p by targeting FOXM1 in non-small cell lung cancer. Cell Physiol Biochem 2016;38:1435-1446.

-26 Cortinovis D, Monica V, Pietrantonio F, Ceresoli GL, La Spina CM, Wannesson L: MicroRNAs in non-small cell lung cancer: current status and future therapeutic promises. Curr Pharm Des 2014; 20:3982-3990.

27 Boeri M, Pastorino U, Sozzi G: Role of microRNAs in lung cancer: microRNA signatures in cancer prognosis. Cancer J 2012;18:268-274.

28 Vannini I, Fanini F, Fabbri M: MicroRNAs as lung cancer biomarkers and key players in lung carcinogenesis. Clin Biochem 2013;46:918-925.

29 Shrestha A, Mukhametshina RT, Taghizadeh S, Vásquez-Pacheco E, Cabrera-Fuentes H, Rizvanov A, Mari B, Carraro G, Bellusci S: MicroRNA-142 is a multifaceted regulator in organogenesis, homeostasis, and disease. Dev Dyn 2017;246:285-290.

-30 Ma Z, Liu T, Huang W, Liu H, Zhang HM, Li Q, Chen Z, Guo AY: MicroRNA regulatory pathway analysis identifies miR-142-5p as a negative regulator of TGF- $\beta$ pathway via targeting SMAD3 Oncotarget 2016;7:71504-71513.

-31 Zhang X, Yan Z, Zhang J, Gong L, Li W, Cui J, Liu Y, Gao Z, Li J, Shen L, Lu Y: Combination of hsa-miR-375 and hsa-miR-142-5p as a predictor for recurrence risk in gastric cancer patients following surgical resection. Ann Oncol 2011;22:2257-2266.

-32 Lou K, Chen N, Li Z, Zhang B, Wang X, Chen Y, Xu H, Wang D, Wang H: MicroRNA-142-5p Overexpression Inhibits Cell Growth and Induces Apoptosis by Regulating FOXO in Hepatocellular Carcinoma Cells. Oncol Res 2017;25:65-73.

33 Zhu J, Zhang Y, Zhang W, Zhang W, Fan L, Wang L, Liu Y, Liu S, Guo Y, Wang Y, Yi J, Yan Q, Wang Z, Huang G: MicroRNA-142-5p contributes to Hashimoto's thyroiditis by targeting CLDN1 J Transl Med 2016;14:166.

34 Tsang FH, Au SL, Wei L, Fan DN, Lee JM, Wong CC, Ng IO, Wong CM: MicroRNA-142-3p and microRNA-142$5 \mathrm{p}$ are downregulated in hepatocellular carcinoma and exhibit synergistic effects on cell motility. Front Med 2015;9:331-343

-35 Chang F, Lee JT, Navolanic PM, Steelman LS, Shelton JG, Blalock WL, Franklin RA, McCubrey JA: Involvement of PI3K/Akt pathway in cell cycle progression, apoptosis, and neoplastic transformation: a target for cancer chemotherapy. Leukemia 2003;17:590-603.

-36 Osaki M, Oshimura M, Ito H: PI3K-Akt pathway: its functions and alterations in human cancer. Apoptosis 2004;9:667-676.

-37 Nicholson KM, Anderson NG: The protein kinase B/Akt signalling pathway in human malignancy. Cell Signal 2002;14:381-395.

-38 Bader AG, Kang S, Zhao L, Vogt PK: Oncogenic PI3K deregulates transcription and translation. Nat Rev Cancer 2005;5:921-929. 


\section{Cellular Physiology Cell Physiol Biochem 2017;43:2505-2515 and Biochemistry Published \begin{tabular}{l|l} 
DOI 10.159/000484459 \\
Pa 2017 The Author(s). Published by S. Karger AG, Basel
\end{tabular} \\ Wang et al.: MiR-142-5p Suppresses Tumorigenesis in NSCLC}

-39 Parsons R: Phosphatidylinositol 3-kinase inhibitors are a triple threat to ovarian cancer. Clin Cancer Res 2005;11:7965-7966.

40 Liang M, Shi B, Liu J, He L, Yi G, Zhou L, Yu G, Zhou X: Downregulation of miR-203 induces overexpression of PIK3CA and predicts poor prognosis of gastric cancer patients. Drug Des Devel Ther 2015;9:3607-3616.

41 Yu QQ, Wu H, Huang X, Shen H, Shu YQ, Zhang B, Xiang CC, Yu SM, Guo RH, Chen L: MiR-1 targets PIK3CA and inhibits tumorigenic properties of A549 cells. Biomed Pharmacother 2014;68:155-161.

42 Xiao W, Gao Z, Duan Y, Yuan W, Ke Y: Downregulation of miR-19a exhibits inhibitory effects on metastatic renal cell carcinoma by targeting PIK3CA and inactivating Notch signaling in vitro. Oncol Rep 2015;34:739746.

43 Chen K, Zeng J, Tang K, Xiao H, Hu J, Huang C, Yao W, Yu G, Xiao W, Guan W, Guo X, Xu H, Ye Z: miR-490-5p suppresses tumour growth in renal cell carcinoma through targeting PIK3CA. Biol Cell 2016;108:41-50.

44 Wang Y, Tang Q Li M, Jiang S, Wang X: MicroRNA-375 inhibits colorectal cancer growth by targeting PIK3CA. Biochem Biophys Res Commun 2014;444:199-204.

45 El-Daly SM, Abba ML, Patil N, Allgayer H: miRs-134 and -370 function as tumor suppressors in colorectal cancer by independently suppressing EGFR and PI3K signalling. Sci Rep 2016;6:24720.

46 Lang Q, Ling C: MiR-124 suppresses cell proliferation in hepatocellular carcinoma by targeting PIK3CA. Biochem Biophys Res Commun. 2012;426:247-252.

47 Liang H, Wang R, Jin Y, Li J, Zhang S: MiR-422a acts as a tumor suppressor in glioblastoma by targeting PIK3CA. Am J Cancer Res 2016;6:1695-707.

48 Liu J, Li Q, Li R, Ren P, Dong S: MicroRNA-363-3p inhibits papillary thyroid carcinoma progression by targeting PIK3CA. Am J Cancer Res 2017;7:148-158.

49 Shi ZC, Chu XR, Wu YG, Wu JH, Lu CW, Lü RX, Ding MC, Mao NF: MicroRNA-375 functions as a tumor suppressor in osteosarcoma by targeting PIK3CA.Tumour Biol 2015;36:8579-8584. 